\title{
An introduction of a novel group theorem to Abstract Algebra
}

\author{
Dillip Kumar Dash ${ }^{1}$, Nduka Wonu' ${ }^{2 *}$ \\ ${ }^{I}$ Fakir Mohan University, Balasore, Odisha, India. \\ ${ }^{2}$ Department of Mathematics/Statistics, Ignatius Ajuru University of Education, Port Harcourt, Nigeria.
}

*Corresponding Author:Nduka WonuPhD, Department of Mathematics/Statistics, Ignatius Ajuru University of Education, Port Harcourt, Nigeria.

\begin{abstract}
This paper proposes a group theorem in Abstract Algebra which is aimed at interestingly showcasing an undiscovered Mathematical concept, using the concept of Euler's Phi Function. The theorem states that: "for all $n \geq 3$ ' $n$ ' divides the sum of all elements of $U(n)$, where is the set of all positive integers less than ' $n$ ' and it is relatively prime to ' $n$ '.'. We also presented a logical proof to the theorem with a few examples in the fora clear understanding of the concept.
\end{abstract}

Keywords: Groups, Abstract Algebra, Euler's Phi Function

\section{INTRODUCTION}

The branch of Mathematics 'Abstract Algebra' consist of a part called 'Group Theory' whose small part describes the set $U(n)$ "The set of all integers less than $n$ and is relatively prime to $n$ such that for all, $k \in U(n)$ g.c.d. $(k, n)=1$ where $n$ belongs to the set of positive integers, that is, $n \in Z^{+} . U(n)$ is a group under multiplication modulo ' $n$ '. The group of units $\mathrm{U}(\mathrm{n})$ is a common groupstudied in an introductory Abstract Algebra class. As far as the above concepts it is easy to verify that one may choose any $n \in N$ or any $n \in Z^{+}$and $U(n)$ will be a group. However, in this concept, we shall deal with only those values of $n$ which are greater than or equal to 3 . But the values $n=1$ and 2 are neglected because this leads to the contradiction of the theorem. Hence, the theorem is valid for only $n \geq 3$. However, the work of [1] contains the example which gives more information about the set $U(n)$. The information about Euler Phi function was also considered in a corollary. More information regarding group theory can be found $[2,3,4]$.

\subsection{Definition}

If $U(n)$ is the set of all prime numbers less than $n$.

In general, the sum of all elements of $U(n)$ (or sum of all prime factors of $n$ ) is given by

$$
\sum_{n=1}^{p-1} U(n)=\frac{n}{2}(n-1), \quad \text { where } n \text { are prime and } n>2
$$

\subsection{Remark}

Note that these elements of $\sum_{n=1}^{p-1} U(n)$ are $3,5,7,9, \ldots, n-1$

\subsection{Does the sum of prime factors of $\boldsymbol{n}$ divide $\boldsymbol{n}$}

In $U(n)$ for instance,

for $n=3$, we have

$U(3)=3$ which is divisible by $n=3$. That is $3 \mid 3=1+2$

For $n=4$, we have

$U(4)=4$ which is divisible by $n=4$. That is $4 \mid 4=1+3$

For $n=5$, we have

$U(5)=10$ which is divisible by $n=5$. That is $5 \mid 10=1+2+3+4$

For $n=6$, we have

International Journal of Scientific and Innovative Mathematical Research(IJSIMR)Page 4 
$U(6)=6$ which is divisible by $n=6$. That is $6 \mid 6=1+5$

For $n=7$, we have

$U(7)=21$ which is divisible by $n=7$. That is $7 \mid 21=1+2+3+4+5+6 .$. .

$U\left(k_{j}\right)=\sum_{i=1}^{j-1} k_{i}$

Which is divisible by $k_{j}$. That is

$$
k_{j} \text { divides } U\left(k_{j}\right)=\sum_{i=1}^{j-1} k_{i}
$$

$j$ (which is the number of elements in the set) has to be even for the division to be possible:To see how true this is, notice that for the equations (2), (3), (4), (5) and (6), we have that $j$ are respectively $2,2,4,2$ and 6 which are all even.

Hence, for all $n \geq 3$ ' $n$ ' divides the sum of all elements of $U(n)$.

\section{THEOREM}

"If $n$ is an integers, the sum of the prime factors of $n$ divides $n$

\section{Proof:}

The proof is already as given in the illustration of section 1.3

(Proved)

\section{CONCLUSION}

The paper is based on the principle that the sum of all the elements of the set $U(n)$ is divisible by $n$ if and only if $n \geq 3$ and $n$ belongs to the set of all positive integers

\section{REFERENCES}

[1] Gillian, J.A (2012). Cotemporary Abstract Algebra,Cengage Learning $8^{\text {th }}$ edition. Retrieved, $23^{\text {rd }}$ of May, 2020 from the web: https://people.clas.ufl.edu/cmcyr/files/Abstract-Algebra-Text_Gallian-e8.pdf

[2] Bialostocki, A (1998). An Application of Elementary Group Theory to Central Solitaire, the College Mathematics Journal, May 1998: 208-212.

[3] White,J.E(1967). Introduction to Group Theory for Chemists, Journal of Chemical Education44 (1967): 128-135.

[4] Herstein, I.N (1975).Topics in Algebra.Wiley Student Edition $2^{\text {nd }}$ edition. Retrieved, $23^{\text {rd }}$ of May, 2020 from the web: https://marinazahara22.files.wordpress.com/2013/10/i-n-herstein-topics-in-algebra-2nd-edition-1975wiley-international-editions-john-wiley-and-sons-wie-1975.pdf

Citation: Dillip Kumar Dash \& Nduka Wonu, (2020). “An introduction of a novel group theorem in Abstract Algebra”. International Journal of Scientific and Innovative Mathematical Research (IJSIMR), 8(3), 4-5. http://dx.doi.org/10.20431/2347-3142.0803002

Copyright: @ 2020 Authors. This is an open-access article distributed under the terms of the Creative Commons Attribution License, which permits unrestricted use, distribution, and reproduction in any medium, provided the original author and source are credited. 